\title{
Aprendiendo a ayudar a aprender. Historia de 10 años de formación de tutores de residentes en Galicia
}

\author{
J.A. Garrido-Sanjuána , M.A. García-Álvarez ${ }^{\text {b }, ~ A . ~ I g l e s i a s-O l l e r o s ~ c, ~}$ \\ S. Lago-Canzobre ${ }^{d}$, F.I. Lago- Deibe e, J.L. Ramil-Hermida ${ }^{\dagger}$
}

Introducción. La formación de los tutores de residentes se ha configurado como uno de los ejes sobre los que recae la calidad del proceso formativo de los especialistas sanitarios. Objetivo. Describir y analizar las características de las acciones de formación de tutores en los 10 últimos años en Galicia, como base del aprendizaje reflexivo para la mejora. Materiales y métodos. Revisión de los programas, contenidos, materiales utilizados, características y procedencia de los participantes, contenidos de los proyectos docentes elaborados y encuestas de valoración. Resultados. Se describen los datos de las 8 ediciones del 'curso de metodología docente y de evaluación para tutores'. Realizaron la formación 86 facultativos. La distribución de participantes por especialidades y áreas sanitarias no se corresponde con el peso de éstas en el sistema asistencial y docente. Las especialidades con más tutores participantes fueron Medicina Familiar y Comunitaria, Anestesiología y Obstetricia-Ginecología. Los 10 facultativos que participaron como docentes estaban en ese momento en ejercicio de su responsabilidad en formación de residentes. Entre los contenidos de los proyectos docentes desarrollados destacan cuantitativamente los dedicados a mejorar la organización de rotaciones o la adaptación de los programas de la especialidad a los centros o unidades, y los dedicados a la mejora metodológica del aprendizaje de habilidades. Se incorporaron nuevas herramientas de evaluación formativa aprendidas durante el curso a muchos de estos proyectos. En las encuestas de valoración final, el curso fue valorado satisfactoriamente por los alumnos, considerando que su contenido era útil para su actividad profesional. Conclusiones. El conocimiento de las áreas sanitarias y especialidades en que ha existido menos incorporación a esta formación permite tenerlo en cuenta a la hora de diseñar acciones futuras. El aprendizaje de nuevas herramientas de evaluación formativa permitió su incorporación a los proyectos docentes. Los alumnos consideraron que el contenido del curso era útil para su actividad profesional.
Palabras clave. Educación médica. Especialidades médicas. Evaluación educacional. Formación de residentes.

\section{Learning to help to learn. The history of 10 years of resident tutors' training in Galicia}

Introduction. The training of the tutors of residents has been formed as one of the axes on which there relapses the quality of the formative process of the medical specialists. Aim. To describe and to analyze the characteristics of the activities of tutors' training in last 10 years in Galicia, as the basis for reflective learning for improvement. Materials and methods. A review of programs, contents, materials, characteristics and origin of the participants and content of teaching projects elaborated. Results. We describe the information of the 8 editions of the 'course of teaching and evaluation methodology for tutors.' 86 professionals achieved this training. The participants' distribution by specialties and health areas does not match the weight of those in the health care and the training systems. The specialties with more tutors participants were Familiar and Communitary Medicine, Anesthesiology and Obstetrics-Gynaecology. The 10 clinic professionals who took part as teachers were at that time in exercising their responsibility in residents' training. Among the contents of the teaching developed projects, stand out quantitatively those focused on improving the organization of rotations or to adapt programs of the specialty to the centers or units, and those devoted to the methodological improvement of learning skills. New formative assessment tools learned during the course were incorporated into many of these projects. In the final questionnaire the course was reported positively by the participants and the contents were considered useful for their professional activity. Conclusions. To know the health areas and specialties in which incorporation to this training has been
Servicio de Medicina Interna. Área Sanitaria de Ferrol.

b Servicio de Psiquiatría. Complejo Hospitalario Universitario de Santiago. Servicio de Medicina Interna. Hospital Juan Canalejo. A Coruña.

d Área Sanitaria de Ferrol.

e Unidad Docente de Medicina Familiar y Comunitaria. Vigo, Pontevedra.

Unidad Docente de Medicina Familiar y Comunitaria. Ferrol, A Coruña, España.

Correspondencia Dr. Juan A. Garrido Sanjuán. Servicio de Medicina Interna. Área Sanitaria de Ferrol. Avda. de la Residencia, s/n. E-15405 Ferrol (A Coruña).

Fax

+34981334529

E-mail

ja_garrido@terra.es

Agradecimientos A J.M. Fornells y su equipo de docentes y a J.M. Manso, que nos empezaron a enseñar cómo trasmitir el 'aprender a aprender' y cuyos materiales han servido de base para el diseño de los cursos que aquí se describen. A F.J. Suso, que coordinó la primera edición de estos cursos. A las personas que desde la Subdirección Xeral de Planificación fueron y siguen siendo sensibles 
a la necesidad de la

formación de tutores para la mejora en la calidad de la formación especializada. A la FEGAS, por su soporte organizativo.

Dedicatoria

A Félix Carrasco, tutor que inició con nosotros como docente esta historia, recientemente fallecido. smaller allows take it into account at the moment of designing future actions. Learning new formative assessment tools led to incorporate them to the final projects. The participants reported the course was useful for their professional activity.

Key words. Educational measurement. Medical education. Medical specialties. Residents' training.

\section{Introducción y objetivos}

La formación especializada está inmersa en un profundo cambio generado por las limitaciones que se han originado de las escasas modificaciones introducidas en el método utilizado para dicha formación desde el comienzo del sistema MIR. Este hecho contrasta con los grandes cambios que se han producido en estos 30 últimos años en la práctica de la medicina.

Hay dos motores esenciales de este cambio en la formación especializada. Por un lado el reconocimiento y potenciación del papel de los tutores, especialmente, y del resto de los participantes en las acciones docentes. Por otro, la mejora en el proceso de evaluación formativa. Ambos motores están vinculados por el desarrollo de una mejora en la metodología docente, potenciando el cambio hacia una enseñanza centrada en el residente. Dicha mejora metodológica debe estar mediada por la formación de los docentes [1], especialmente de los tutores, en dichos métodos, y por otro lado es la que debe generar el cambio conceptual y práctico en el sistema de evaluación.

El sistema MIR ha seguido un procedimiento de aprendizaje esencialmente 'oportunístico', aprovechando las ocasiones de aprendizaje que proporciona la asistencia sanitaria habitual $[1,2]$. Sin embargo la forma de afrontar estas oportunidades docentes sí puede programarse metodológicamente de forma que cuando surjan se obtenga de ellas un mayor aprendizaje. Esto tiene especial importancia en un sistema que por su actual regulación $[3,4]$ ha supuesto una reducción en los tiempos de actividad asistencial que los residentes realizan.

Los modelos docentes centrados en la persona que aprende y las estrategias de aprender a aprender se centran en la reflexión. La reflexión sobre la acción [5], sobre lo realizado, sobre como se han abordado esas oportunidades docentes, para detectar lo que no sabemos hacer adecuadamente
[6] y como podemos adquirir ese conocimiento, individualizándolo para el alumno concreto, se considera la base del método del aprendizaje del adulto [7]. Por ello tiene sentido formativo la recogida de datos de lo realizado para que su análisis nos sirva como punto de partida de la mejora.

El proceso que describimos a continuación surge de la detección de la necesidad de la formación de tutores como eje esencial de los cambios necesarios en el sistema MIR y la demanda, por parte de las comisiones de docencia hospitalaria (CD) y las comisiones asesoras (CA), de una respuesta a esta necesidad. La Administración Sanitaria a través de la Subdirección Xeral de Planificación Sanitaria, sensible a la solicitud y compartiendo esta necesidad, promovió entre los años 1998 y 2000 unos cursos coordinados e impartidos por profesionales del Institut d'Estudis de la Salut de Cataluña (IES). Se animó a incorporarse a estos cursos a tutores de especialidades hospitalarias y de atención primaria y presidentes de las CD y CA de las 7 áreas sanitarias y hospitales/complejos hospitalarios acreditados para la formación especializada. La información y selección de los alumnos se realizó desde las gerencias, CD y CA. Los cursos se impartieron de forma centralizada en Santiago de Compostela, gestionados por la Fundación Pública da Escola Galega de Administración Sanitaria (FEGAS).

El siguiente paso fue el desarrollo de un curso para formación de formadores cuya finalidad fue capacitar a algunos de los tutores y jefes de estudios que habían realizado la formación previa para dar continuidad a la formación del resto de tutores y de aquellos que se fueran incorporando a las labores de tutoría.

El objetivo es describir las características de las acciones de formación de tutores promovidas desde la Subdirección Xeral de Planificación Sanitaria de Galicia, programadas a instancias de las CD y CA de las unidades docentes (UD) de medicina familiar y comunitaria (MFyC) al detectar dicha necesidad. Se comunican las innovaciones, aportaciones o diferencias con los cursos previos que sirvieron de base y se describen las características de los docentes y tutores participantes y los proyectos docentes diseñados. El fin último de la comunicación es ofrecer el análisis de este material y la reflexión sobre el mismo para aquellos que deseen utilizarlos para diseñar y mejorar nuevas acciones formativas. 


\section{Materiales y métodos}

Revisión de los programas, contenidos y materiales utilizados, de la relación de participantes que obtuvieron acreditación por completar la formación estipulada, de los datos recogidos por el programador y coordinador docente del curso y de los títulos de los proyectos docentes presentados al final de los cursos. Los datos referentes a la descripción del curso se relatan como 'resultados' como parte de los datos obtenidos de la revisión. Al final de cada edición del curso la organización (FEGAS), independiente del equipo docente, solicitó a los alumnos que rellenaran una encuesta de valoración de la calidad y satisfacción del curso, que contenía nueve enunciados o afirmaciones relativas al desarrollo de la acción docente, con cinco posibles respuestas según el grado de acuerdo con las afirmaciones.

Se utiliza predominantemente una metodología cualitativa que no pretende comprobar ni rechazar una hipótesis sino que aspira a construir conocimiento a través de los datos. En la presentación de los resultados se utiliza principalmente un método narrativo apoyado en tablas y figuras, poniéndose a disposición de quien los precise otros detalles (programas y cronogramas más concretos, fichas utilizadas, títulos concretos de los proyectos docentes diseñados, etc.). Se narran algunos detalles de la metodología utilizada en el curso como ejemplo de la insistencia que en él se hizo de que si los pequeños detalles de planificación e infraestructura (el por qué de la distribución horaria, de la localización geográfica, forma de anunciar el curso...) no están bien argumentados o justificados metodológicamente pueden hacer fracasar una acción docente.

Aunque actualmente, tras la modificación legislativa sobre la formación especializada [3], se engloban con el término de CD tanto las así denominadas previamente como las antiguas CA, mantendremos esta nomenclatura diferencial que existía en el periodo al que se refiere este trabajo.

\section{Resultados}

\section{Número de cursos y años de desarrollo}

A partir de 2003, en que se realizó el primer curso denominado 'curso de metodología docente y de evaluación para tutores de formación especializada', se han desarrollado ocho ediciones hasta 2008. La descripción de contenidos y participantes del presente trabajo corresponde a estas ocho ediciones. Las tres primeras ediciones se centralizaron en Santiago, diversificándose geográficamente las otras cinco para facilitar el acceso de profesionales locales: Ferrol, Vigo, Coruña, Lugo y Orense. Con la excepción de la primera edición, las otras siete han sido programadas y coordinadas por la misma persona, que sí participó como docente también en la primera.

\section{Objetivos del curso}

En los programas para informar de la puesta en marcha del curso se enunciaba como objetivo general del mismo: 'Mejorar la calidad de la formación especializada a través del aprendizaje de metodología docente, de las bases y técnicas de evaluación formativa y de la delimitación de las funciones tutoriales, por parte de las personas que ejercen las tareas de tutores de los facultativos especialistas en formación' Los objetivos docentes intermedios se recogen en la tabla I. Los objetivos específicos se enunciaron al inicio de cada sesión de trabajo.

\section{Programa y contenidos del curso}

Se trata de un curso presencial de 30 horas. Los contenidos siguieron el esquema básico de los cursos recibidos por parte de los docentes del IES adaptándolos a la realidad de la formación especializada en Galicia y actualizándolos con avances generados por nuevas publicaciones en metodología docente y con contenidos trabajados en la Asociación Nacional de Redes de Comisiones de Docencia y Asesoras (AREDA) y la Red de CD y CA de Galicia (REDEGA) que se han desarrollado durante estos años. Asimismo se fueron introduciendo los contenidos, inicialmente de los borradores y en las dos últimas ediciones de los decretos ya aprobados, de la nueva legislación que regula la formación especializada en el ámbito laboral del residente [4] y en la vertiente docente [3]. Destaca como contenido más novedoso en estas ediciones, respecto a las del IES, la introducción de una sesión de reflexión sobre 'conflictos éticos en el aprendizaje de residentes', cuya metodología y contenidos han sido previamente publicados [2]. Dicha sesión 
Tabla I. Objetivos intermedios.

- Introducir el concepto y los métodos de la enseñanza centrada en quien aprende

- Delimitar y definir las funciones del tutor

- Conocer los medios necesarios para desarrollar la labor tutorial

- Intercambiar la experiencia tutorial de distintas especialidades

- Conocer la figura de 'colaborador docente'

- Definir la evaluación formativa y sus distintas técnicas

- Practicar métodos de evaluación formativa

- Conocer el concepto de objetivos docentes, sus distintos tipos y practicar la forma de enunciarlos

- Capacitar al tutor en la detección de necesidades de aprendizaje, diseño y planificación de propuestas docentes

- Situar la formación de posgrado en su contexto y conocer las Redes de Comisiones de Docencia y Asesoras

- Conocer la nueva legislación que sustenta la actividad laboral y formativa durante el periodo de residencia

se realizaba al final del curso, siendo utilizada como un argumento más para hacer evidente la necesidad de aumento de los medios humanos y materiales para mejorar la formación de los futuros especialistas, especialmente las acciones de aprendizaje que tienen interacción directa con el paciente.

Las principales características de la metodología empleada y los mensajes esenciales se recogen en la tabla II. La frase 'aprender haciendo' resume la esencia de la metodología que se pretendió emplear, recogiéndose en la tabla III las habilidades a aprender que fueron ejercitadas en mayor o menor medida en el curso. Se utiliza a lo largo de este texto con frecuencia la terminología 'acción docente' en lugar de la de 'curso' para resaltar el uso de esta metodología en contraste con la más clásica de exposición de conocimientos por parte del docente, que tradicionalmente se vinculaba al término 'curso'. Para hacer posible esta metodología participativa se limitó el número de alumnos a 14 por edición. Se insistió en que la finalidad no era convertirse en especialistas en metodología docente ni elaborar unas perfectas herramientas
Tabla II. Características esenciales de la metodología del curso y mensajes clave.

- Curso presencial

- Grupo pequeño

- Centrado en el alumno:'lo importante es lo aprendido y no lo enseñado'

- 'Aprender haciendo'

- Aprendizaje a través del ejemplo

- Docentes con ocupación tutorial activa

- Intercambio de experiencias docentes entre áreas de salud y especialidades

- Aun admitiendo el sistema de residencia como un aprendizaje 'oportunístico', se puede programar metodológicamente el abordaje de las 'oportunidades docentes'y suplir las que no han surgido mediante otros métodos (simulación...)

- Evaluación y resumen/conclusiones de cada sesión de trabajo

- Importancia del 'desaprendizaje' para dejar espacio a la innovación

- Importancia de la detección de lo ignorado: el 'no lo sé' como las tres palabras esenciales para el aprendizaje

de evaluación sino desarrollar de forma individual y en los servicios y centros docentes aquellas habilidades y herramientas ofertadas que mejor pudieran aplicarse al objetivo de mejorar la formación de nuestros especialistas.

\section{Cronograma}

Respecto a la forma de distribuir el tiempo del curso destacar que se planificó para que una parte principal del curso se realizara dentro de la jornada laboral (20 horas), si bien seguía incluyendo un esfuerzo significativo de dedicación de tiempo personal (10 horas). Las 30 horas se combinaban en cinco jornadas de seis horas. La quinta jornada estaba separada entre cuatro y ocho semanas de las otras cuatro, para permitir un periodo necesario para el diseño del proyecto docente que era presentado en la última jornada.

Formando parte de la metodología de refuerzo del aprendizaje se solicitaba a los participantes rellenar al inicio del curso un test de respuestas múltiples sobre conceptos de metodología docente y de evaluación, que se repetía al final 


\section{Tabla III. Habilidades practicadas durante} el curso.

- Elaboración/redacción de objetivos docentes

- Identificación de actitudes, habilidades y conocimientos en el enunciado y contenido de objetivos

- Algunos métodos de evaluación formativa: - Feedback

- Self-audit

- Revisión por pares (peer-review)

- Test de preguntas cortas

- Práctica de videograbación

- Identificación del escalón de la pirámide de Miller al que corresponden distintas herramientas de evaluación

- Ejercicio de trabajo en grupo pequeño

- Ejercicio de gestión de conflictos

- Evaluación de contenidos de las sesiones de trabajo y resúmenes en forma 'hoy hemos aprendido...' o'hemos tratado de aprender...'

- Casos simulados de conflicto ético

- Evaluación de los docentes mediante fichas específicas para detección de cualidades, habilidades, conocimientos y actitudes que debe tener un docente

- Diseño de proyecto docente

- Exposición pública de un tema

- Discusión crítica utilizando criterios preestablecidos

del curso. El test inicial incluía como pregunta abierta: 'Enuncia algo que no sabes y que esperas aprender a lo largo del curso'. La pregunta abierta del test de final de curso decía: 'Enumera algo que hayas aprendido. ¿Hay algo que antes no sabías hacer y ahora sí??

\section{Docentes participantes}

El programador y coordinador y todos los docentes participantes en las 8 ediciones que se describen eran jefes de estudios/presidentes de $\mathrm{CD}$ (2), presidentes de CA/coordinadores de UD de MFyC (3), o tutores (5) en activo en su labor tutorial. En cuanto a las especialidades de los mismos, cuatro eran especialistas en MFyC, tres en medicina interna, dos en psicología clínica y uno en cirugía general. Todos habían realizado formación como tutores y ocho de ellos el curso de formación de formadores. Participaron cinco o seis docentes en cada edición.

\section{Coordinación}

Los docentes recibieron previamente los contenidos y presentaciones que iban a utilizar los otros docentes, así como una copia del disco compacto con el material entregado a los alumnos. El programador del curso asistió al menos a una de las sesiones impartidas por el resto de los docentes como medio añadido de coordinar y tratar de disminuir superposición o duplicidad de contenidos.

\section{Materiales utilizados}

Se entregó a los alumnos un disco compacto que incluía los materiales y documentos de trabajo utilizados en el curso así como aquellas publicaciones que estaban disponibles de forma abierta en la red, entre las utilizadas o recomendadas. Asimismo se incluyó algún video docente como ejemplo de este recurso formativo.

Se utilizó una webcam para la realización del ejercicio de videograbación, con grabación directa en formato digital. Se entregó a cada alumno la grabación de su propia participación en la sesión de simulación del ejercicio de feedback. Se utilizaron ejercicios prácticos recogidos en la guía pedagógica para el personal de la OMS [8].

\section{Características de los tutores participantes}

Obtuvieron la certificación del curso 86 profesionales. Se informó a la entidad responsable de la organización y difusión de la información sobre el curso de la no cobertura de inscripciones en dos ediciones y de la no presentación de alumnos inscritos en otras ediciones. Doce de los 86 no eran tutores en ese momento, ya que se incluyeron como destinatarios secundarios del mismo otros 'colaboradores docentes'. Tres eran presidentes de CA y uno de CD. La distribución por áreas sanitarias (incluyendo los hospitales de referencia de dichas áreas) y por áreas de ejercicio de la actividad docente se describe en las figuras 1 y 2 . En la figura 3 se agrupan por especialidades médico-clínicas (23), incluyendo en ellas otras que ejercen su actividad en relación directa con el paciente, como los psicólogos clínicos, quirúrgicas (19) y servicios centrales (11), aquellas sin relación clínica di- 


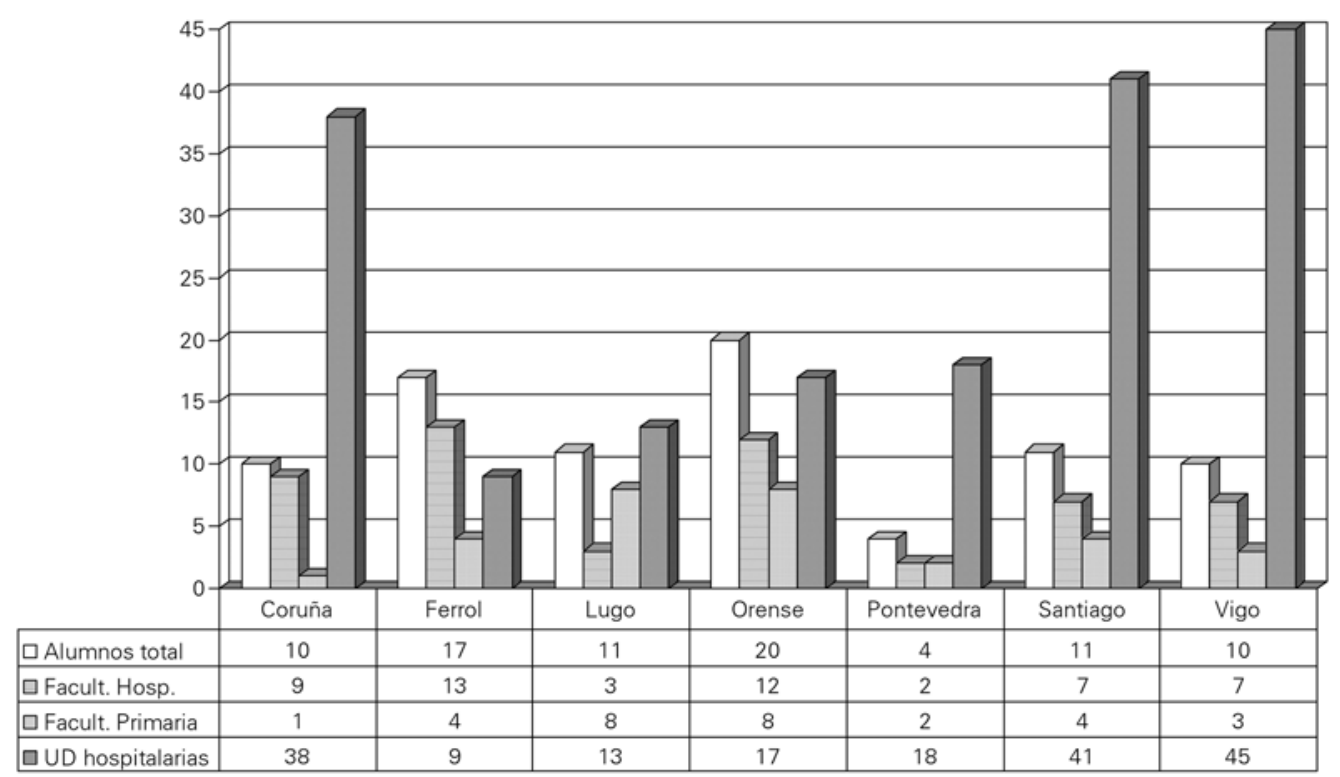

Figura 1. Distribución de los facultativos participantes en los cursos por áreas sanitarias y según el ejercicio preferente de su actividad docente. Comparación con el número de unidades docentes hospitalarias acreditadas.

recta. Destacan como especialidades con mayor número de alumnos participantes en el curso MFyC (33), Anestesiología (7), Obstetricia y Ginecología (6), Análisis Clínicos, Psiquiatría, Medicina Interna y Psicología Clínica (4 cada una). Entre las especialidades deficitarias resaltan, especialmente al compararlo con el número de UD y plazas ofertadas de la especialidad, Traumatología (un solo alumno que no era tutor en el momento del curso, frente a las 14 plazas ofertadas en 9 UD) y Radiología (un tutor participante, frente a las 16 plazas en $8 \mathrm{UD}$ ). Al menos tres personas que hicieron el curso sin ser tutores fueron nombradas posteriormente para dicha actividad.

Los tutores de especialidades hospitalarias debían ser al menos 175 en Galicia en 2007 si suponemos un mínimo de uno por especialidad acreditada y hospital $(76,65$ y 34 según la agrupación definida para la figura 3). Agrupadas las especialidades de los facultativos hospitalarios participantes según se indica en la figura 3 sí mantenían proporcionalidad con las UD existentes de esos grupos de especialidades. Para la convocatoria 2007-2008, en Galicia se ofertaron 247 plazas de facultativos

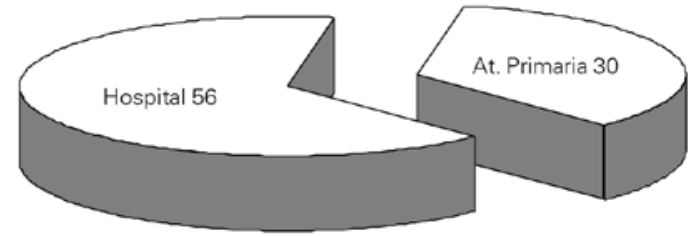

Figura 2. Distribución de los facultativos participantes en el curso según el área de ejercicio de su actividad docente.

para especialidades hospitalarias (107, 95 y 45, agrupándolas según los grupos de la figura 3) y 85 de MFyC.

\section{Requisitos para la certificación del curso}

Además del registro presencial acreditado mediante firma en cada sesión, se requería la realización del diseño de un proyecto docente según los criterios trasmitidos durante el curso (Tabla IV), su exposición en el último día del curso, la participación crítica en la discusión de los pro- 


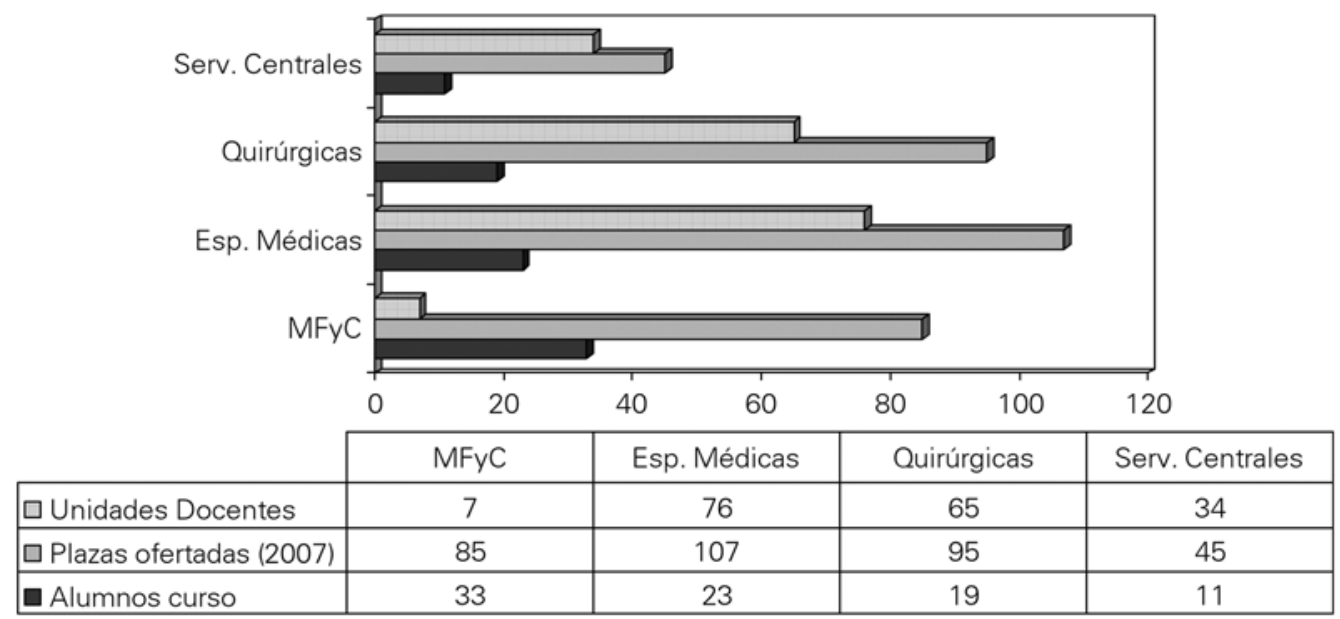

Figura 3. Distribución de los facultativos participantes en el curso según su especialidad, comparando con el número de unidades acreditadas y plazas ofertadas.

Tabla IV. Fases del desarrollo y criterios para el diseño de los proyectos docentes.

- Detección de necesidades de formación y descripción del método utilizado para su detección

- Definición y jerarquización de objetivos

- Sistema y herramientas de evaluación

- Estrategia de planificación: qué, a quién, cómo, medios, en qué contexto, cuándo. .

- Realización práctica

- Evaluación formativa y de resultados. Eficiencia, impacto

yectos del resto de alumnos, entregar las fichas de valoración de los docentes y realizar el test previo y el test final del curso.

\section{Proyectos docentes de final de curso}

Los contenidos de los proyectos presentados aparecen en la tabla $\mathrm{V}$ distribuidos en 9 grupos. La puesta en marcha de herramientas de evaluación formativa tipo self-audit o feedback fue utilizada en varios de los proyectos de los grupos 2 y 5 . En el grupo 2 se incluyen trabajos que protocolizaron procesos asistenciales. El grupo 1 incluye entre el subgrupo de MFyC aquellos proyectos de organización vinculados a rotaciones de residentes de MFyC en el hospital. La asistencia de patología urgente ocupó al menos nueve de los proyectos (dentro de los grupos 1 y 2). En el grupo 9 (otros) destacan dos proyectos vinculados a la mejora de la asistencia en atención primaria a las personas afectadas por violencia de género.

Los proyectos docentes permitieron una aproximación a corto plazo a la eficacia de la acción docente, a través de la verificación de su adhesión a los criterios preestablecidos (Tabla IV), especialmente la obligación de diseñar métodos de evaluación vinculados a los objetivos, y la incorporación de herramientas de evaluación aprendidas a varios de los proyectos.

\section{Encuestas de valoración del curso por los alumnos}

Se dispone de resultados de 78 encuestas finales de valoración del curso por los alumnos, que se presentan en la tabla VI. Un 96\% de los que respondieron estaban 'de acuerdo' o 'muy de acuerdo' con la afirmación de que el curso les pareció bueno globalmente y con la de que el contenido del curso les es útil para su actividad profesional.

En la pregunta abierta del test final los alumnos enumeraron habilidades o destrezas que consideraban habían adquirido o aprendido durante el curso. 
Tabla V. Contenido de los proyectos docentes presentados en los cursos.

\begin{tabular}{lc}
\hline 1. Organización de rotaciones o adaptación de programas de la especialidad & 18 \\
\hline MFyC (5 relacionados con atención urgencias) & 7 \\
\hline Resto de especialidades & 11 \\
\hline 2. Acciones de mejora de procedimientos diagnóstico-terapéuticos (4 vinculados a asistencia de urgencias) & 14 \\
\hline MFyC & 7 \\
\hline Resto de especialidades & 7 \\
\hline 3. Centrados en aprendizaje de habilidades (talleres, simuladores...) & 14 \\
\hline 4. Organización de sesiones, cursos, actividades de formación continuada, etc. & 11 \\
\hline 5. Mejora de elaboración de historia clínica y/o exploración física & 6 \\
\hline 6. Aprendizaje de conocimientos teóricos & 5 \\
\hline 7. Mejora de consultas monográficas o interconsultas (y elaboración informes) & 4 \\
\hline 9. Aprendizaje de utilización de guías de práctica clínica y toma de decisiones basada en evidencias & 3 \\
\hline
\end{tabular}

\section{Discusión}

Uno de los puntos esenciales para que una acción docente sea adecuada es que se haya generado para solucionar una necesidad formativa detectada [9]. Hablamos de pertinencia para denominar esta característica esencial de los objetivos docentes: su coherencia con la necesidad que les dio origen $[10,11]$. El que la acción docente sea capaz de cubrir esa deficiencia es el mejor y verdadero criterio de evaluación de la eficacia de la misma. Las acciones que aquí se describen fueron generadas por una necesidad sentida y vivida por aquellos que han sido los destinatarios de los mismos. Esto también ha tenido impacto en el grado de participación e implicación activa de los alumnos que se inscribieron, a diferencia de acciones que pueden ser realizadas únicamente para completar un currículo o conseguir la acreditación oficial vinculada al curso. Como dice
Saura-Llamas [12], tutor efectivo es el que, entre otras características, conoce lo que necesita para formar residentes. La afirmación mayoritaria de los alumnos en la encuesta final de valoración de que los contenidos eran útiles para su actividad profesional reafirma la pertinencia de la acción docente. Es de destacar esta afirmación porque implica además que los alumnos tenían asumida o asumieron a partir del curso la actividad tutorial como parte de su actividad profesional, hecho trascendente en el camino emprendido hacia la profesionalización de la acción tutorial.

El curso trata de que los tutores aprendan a ayudar a aprender a los residentes poniendo la reflexión en el centro del método de aprendizaje. Esta comunicación pretende centrarse en esa misma estrategia: reflexionar sobre lo desarrollado para que esta información tenga utilidad de mejora formativa al ser devuelta a los que la generaron y que están en el propio proceso de 
Tabla VI. Encuestas de valoración final del curso por los alumnos $(n=78)$.

\begin{tabular}{|c|c|c|c|c|}
\hline & Muy de acuerdo & De acuerdo & Fue normal & En desacuerdo \\
\hline $\begin{array}{l}\text { El curso le proporcionó conocimientos } \\
\text { adecuados y actualizados }\end{array}$ & $33(42,31 \%)$ & $43(55,13 \%)$ & $2(2,56 \%)$ & $0(0,00 \%)$ \\
\hline El número de horas del curso fue el adecuado & $22(28,21 \%)$ & $44(56,41 \%)$ & $3(3,85 \%)$ & $8(10,26 \%)$ \\
\hline $\begin{array}{l}\text { El contenido del curso es útil } \\
\text { para su actividad profesional }\end{array}$ & $40(51,28 \%)$ & $35(44,87 \%)$ & $3(3,85 \%)$ & $0(0,00 \%)$ \\
\hline $\begin{array}{l}\text { El material y la documentación } \\
\text { docente le parecieron adecuados }\end{array}$ & $40(51,28 \%)$ & $35(44,87 \%)$ & $3(3,85 \%)$ & $0(0,00 \%)$ \\
\hline $\begin{array}{l}\text { La coordinación de las materias expuestas } \\
\text { entre los/as relatores/as fue buena }\end{array}$ & $28(35,90 \%)$ & $41(52,56 \%)$ & $9(11,54 \%)$ & $0(0,00 \%)$ \\
\hline $\begin{array}{l}\text { El perfil profesional de los } \\
\text { asistentes fue adecuado }\end{array}$ & $35(44,87 \%)$ & $39(50,00 \%)$ & $4(5,13 \%)$ & $0(0,00 \%)$ \\
\hline $\begin{array}{l}\text { La atención al alumnado por } \\
\text { la organización fue correcta }\end{array}$ & $24(30,77 \%)$ & $38(48,72 \%)$ & $16(20,51 \%)$ & $0(0,00 \%)$ \\
\hline Las condiciones de los locales fueron idóneas & $24(30,77 \%)$ & $39(50,00 \%)$ & $13(16,67 \%)$ & $2(2,56 \%)$ \\
\hline Globalmente el curso le pareció bueno & $36(46,15 \%)$ & $39(50,00 \%)$ & $1(1,28 \%)$ & $0(0,00 \%)$ \\
\hline
\end{tabular}

aprendizaje (feedback), o, como en este caso, ser trasmitida a otros que quieran iniciar o mejorar procesos de aprendizaje similares.

En algunas áreas de nuestra actividad profesional (ética, comunicación, docencia, etc.), aunque es deseable que trabajemos por aumentar los trabajos con metodología prospectiva y comparativa con grupos control, buscando asentar evidencias, hay que reconocer el valor, conviviendo con dichas investigaciones, de los métodos cualitativos y narrativos. Éstos han aportado y pueden seguir aportando datos que construyan conocimiento y, sobretodo, que sirvan de base a la reflexión como forma de aprendizaje. Como tenemos pocos datos que nos permitan hablar de evidencias sobre efectividad docente, tenemos que utilizar datos del proceso, asumiendo inicialmente que si mejoramos el proceso docente obtendremos resultados mejores, es decir, mejoraremos la calidad de nuestros especialistas [12].
Cuando se pretende mejorar áreas que llevan años siguiendo la misma metodología, es importante la capacidad de 'desaprender' de los que van a intervenir en ese cambio. Se entiende en este caso por desaprender la capacidad de dejarse interpelar por nuevas estrategias y métodos, sin sentirse atados a los métodos clásicamente utilizados. En este caso las estrategias de aprendizaje centradas en el residente y en como ayudar a aprender, obligan a los tutores a reconocer las limitaciones de los métodos clásicos, más expositivos y centrados en el docente, y reducir su utilización para dar cabida a la nueva estrategia. Se hizo hincapié en ello en el curso, pero resaltando también la necesidad de reubicar estrategias previas que han sido claramente válidas hasta la actualidad, haciéndolas convivir con las nuevas estrategias. Esos métodos previos pueden ser, por ejemplo, los más adecuados para cubrir las lagunas en la adquisición de los conocimientos o bases teóricas de las especialidades por los 
residentes actuales (el primer escalón, la base, de la pirámide de Miller [13]).

Sin minusvalorar en absoluto, sino muy al contrario como ya ha quedado reflejado, el papel de los especialistas en metodología docente, sí queremos destacar dentro de las innovaciones docentes, el que la coordinación y peso docente del curso haya correspondido a personas que estaban en el día a día en contacto con los residentes. Esto supone un valor añadido al permitir un acercamiento actualizado a los problemas de la formación sanitaria especializada in vivo y una cercanía a la comprensión de las dificultades que los alumnos tutores manifestaban en cuanto al traslado a la práctica asistencial diaria de lo allí aprendido. Fue un elemento facilitador de la reflexión sobre la realidad vivida en las UD y la forma de integrar lo aprendido.

El cronograma se estableció para permitir que la mayoría de horas del curso se dieran dentro de la jornada laboral, como forma de reconocer el esfuerzo que ya los tutores realizan en su jornada para las labores de tutoría y de reivindicar el reconocimiento de tiempos para estas labores y su formación vinculada. Sin embargo, esto generó dificultades para que otros tutores asistieran, para que algunos de los asistentes completaran las horas y algunos tutores reconocieron que parte $\mathrm{o}$ todo el tiempo del curso lo sacaron de sus días libres o salidas de guardia. La nueva legislación que regula el sistema de formación especializada $[3,4]$ debe ayudar a que en los centros exista obligación, si quieren seguir manteniendo su acreditación docente, de programar tiempos de dedicación tanto a la labor tutorial como a la formación en metodología docente dentro de la jornada laboral [14]. Supone un consumo de recursos para el sistema que es necesario para garantizar la calidad de la asistencia que la sociedad tiene que demandar al Estado, con implicaciones éticas [2,15]. Se puso un especial énfasis en la enseñanza mediante el 'ejemplo' o 'modelo' como forma de aprendizaje para resaltar su importancia en la formación del residente $[2,16]$. Se marcó la importancia de este tema mediante la utilización de una ficha que desglosa los aspectos a evaluar en un docente, que fue rellenada de forma anónima por los alumnos respecto a todos los docentes participantes. En esta misma línea metodológica se propuso a los alumnos el ejercicio de recuerdo de un docente que hubiera dejado especial huella para ellos a lo largo de su vida, para después deducir a partir de las características de los mismos las cualidades que debería tener un buen docente. Especialmente en el campo del aprendizaje de las actitudes, el tutor tiene que ser consciente del papel que como 'modelo' va a ejercer sobre el residente.

La falta de correlación entre el número de tutores o colaboradores docentes participantes procedentes de cada área sanitaria y el número de especialistas en formación, tutores y población atendida, puede estar en relación con la diferente forma en que llegara la información y el estimulo y facilidades proporcionadas para la incorporación al curso en las distintas áreas. Asimismo pudo estar influida por el número de tutores que tuvieran ya formación por participación previa en otras acciones docentes para tutores proporcionadas por otras entidades y organizaciones o en las ediciones previas impartidas por el IES. Sin embargo no se han investigado estas distintas hipótesis, limitándose el trabajo a describirlo. Llama la atención en este sentido el predominio de participantes de las áreas más pequeñas con menor número de plazas acreditadas (Ferrol, Lugo, Orense). Es una excepción Pontevedra, que fue la única en que no se hizo una edición local. La detección de áreas sanitarias y especialidades (datos pormenorizados no presentados) en que esta formación ha sido menos desarrollada permite buscar las causas y poner medios de estimulo o facilitación de dicha formación en esos ámbitos, siendo ésta otra de las aportaciones de este trabajo.

Al menos tres de los facultativos que no eran tutores en el momento de realizar el curso accedieron a esta labor con posterioridad. Probablemente esa formación, vinculada en sí misma al interés por la docencia, facilitó el acceso al nombramiento.

Entre los contenidos elegidos para la elaboración de los proyectos docentes de final de curso llama la atención cuantitativamente el aprovechamiento de los mismos para organizar y adaptar programas y rotaciones, así como la importancia dada a la estructuración del aprendizaje y evaluación de la adquisición de habilidades y a la mejora de la historia clínica y exploración física. Algunos de los alumnos al presentar sus proyectos destacaron la necesidad de recuperar el aprendizaje y estudio de las bases 'teóricas' de la especialidad, minusvaloradas a veces por los residentes. Destaca también la preocupación de los tutores por la mejora del aprendizaje de la asistencia a la pato- 
logía urgente, reflejada por el número de proyectos que se centraron en este tema.

La eficacia de acciones docentes similares suele medirse a corto plazo, al finalizar el curso, si bien hay en la literatura cada vez más intentos de buscar medidas del impacto trascurrido un tiempo después del curso $[17,18]$. Los métodos utilizados para medir este impacto suelen consistir en cuestionarios de autoevaluación que pretenden recoger la percepción por parte de los propios alumnos de cómo ha influido el curso en su forma de llevar a cabo su función docente. Tratan de detectar el grado de introducción de nuevas herramientas o habilidades docentes en su práctica docente habitual [18]. En este trabajo tenemos tres acercamientos a medir el impacto a corto plazo del curso. Dos están determinados por la propia percepción referida por los alumnos: expresión de un alto grado de acuerdo en que los contenidos del curso les eran útiles para su actividad profesional y enumeración de contenidos percibidos como aprendidos en la pregunta abierta del test final. El tercero es la incorporación en sus proyectos finales de una sistemática planteada en el curso a la hora del diseño de las acciones docentes y la inclusión de herramientas de evaluación formativa trabajadas en el curso. La elaboración del proyecto podría considerarse un método de evaluación de los niveles 2-3 de Miller de competencia tutorial $[13,19]$. Es deseable en acciones futuras diseñar métodos de medida del impacto a medio y largo plazo $[17,18]$.

Una de las finalidades de la recopilación de la información utilizada en el curso en formato digital, además de simplificar su archivo y trabajo posterior por los alumnos, es facilitar que pudiera ser utilizada en sus centros para incorporar a la formación en metodología a otros tutores y colaboradores docentes.

Es importante que los expertos en docencia nos ayuden a los clínicos, a los que estamos en el día a día con el residente, y aprendamos de ellos y con ellos. Sin embargo no debemos caer en el riesgo de convertir el perfeccionar el instrumento docente o de evaluación en el centro de la acción. El perfeccionamiento del método no puede convertirlo en complejo, farragoso y, sobre todo, no puede convertirse en un competidor con nuestro verdadero objetivo que es que los residentes aprendan.

Concluimos que acciones docentes específicas para la formación de tutores de residentes con contenidos y métodos que favorecen el aprendizaje mientras se practica y la reflexión sobre lo realizado son bien valorados por éstos, considerándolas de utilidad para su actividad profesional.

\section{Bibliografía}

1. Millán J, García-Seoane J, Calvo-Manuel E, Díez-Lobato R, Calvo-Manuel F, Nogales-Espert A. Relaciones de la facultad de medicina con el hospital universitario. La enseñanza de la medicina clínica. Educ Med 2008; 11 : 3-6.

2. Garrido JA. Conflictos éticos en el aprendizaje de los residentes. An Med Interna 2006; 23: 493-502.

3. Real Decreto $183 / 2008$, de 8 de febrero, por el que se determinan y clasifican las especialidades en ciencias de la salud y se desarrollan determinados aspectos del sistema de formación sanitaria especializada. BOE n. ${ }^{\circ} 45$, de 21 de febrero de 2008.

4. Real Decreto $1146 / 2006$, de 6 de octubre, por el que se regula la relación laboral especial de residencia para la formación de especialistas en Ciencias de la Salud. BOE n. ${ }^{\circ} 240$, de 7 de octubre de 2006.

5. Brookfield S. Critically reflective practice. J Contin Educ Health Prof 1998; 18: 197-205.

6. Editor's choice. 'I don't know': the three most important words in education. BMJ 1999; 318: 1223.

7. Fornells JM, Julià X, Arnau J, Martínez-Carretero JM. Feedback en educación médica. Educ Med 2008; 11: 7-12.

8. Gilbert JJ. Guía pedagógica para el personal de salud [ed. española]. Valladolid: Universidad de Valladolid; 1994.

9. García-Barbero M. Planificación educativa en ciencias de la salud. Barcelona: Masson-Salvat; 1995.

10. Manso JM. ¿Qué enseñar en ciencias de la salud? Técnicas para definir competencias y perfiles profesionales (1. a parte). Educ Med 2000; 3: 61-8.

11. Simpson MA. Objections to objectives. Med Teach 1980; 2: 229-31.

12. Saura-Llamas J. Cómo puede convertirse un tutor en un docente efectivo. Aten Primaria 2007; 31: 151-5.

13. Miller GE. The assessment of clinical skills/competence/ performance. Acad Med 1990; 65 (Suppl 9): S63-7.

14. Álvarez JA, Vicent MD, Salamanca JM, Pérez F, Carrasco M. El tutor y la tutoría en el proceso de formación de especialistas sanitarios en la comunidad de Madrid. Análisis e interpretación mediante grupos focales. Educ Med 2003; 6: 100-11.

15. AREDA (Asociación de Redes de Comisiones de Docencia y Asesoras). La evaluación de la formación especiali- 
zada como garantía de la calidad del sistema de salud. Educ Med 2007; 10: 16-25.

16. Bligh J. Learning by example. Med Educ 1999; 33: 234-5.

17. Godfrey J, Dennick R, Welsh C. Training the trainers: do teaching courses develop teaching skills? Med Educ 2004; 38: 844-7.
18. Rubak S, Mortensen L, Ringsted C, Malling B. A controlled study of the short- and long-term effects of a Train the Trainers course. Med Educ 2008; 42: 693-702.

19. Lago-Deibe F, Ferreiro-Gurí JA. Portafolio: una herramienta de evaluación formativa para el médico de familia (I). Cad Aten Primaria 2006; 13: 95-100. 